\title{
Modeling Dwell Time Engagement on Visual Multimedia
}

\author{
Hemank Lamba* \\ Carnegie Mellon University \\ hlamba@cs.cmu.edu
}

\author{
Neil Shah \\ Snap Inc. \\ nshah@snap.com
}

\begin{abstract}
Visual multimedia is one of the most prevalent sources of modern online content and engagement. However, despite its prevalence, little is known about user engagement with such content. For instance, how can we model engagement for a specific content or viewer sample, and across multiple samples? Can we model and discover patterns in these interactions, and detect outlying behaviors corresponding to abnormal engagement? In this paper, we study these questions in depth. Understanding these questions has implications in user modeling and understanding, ranking, trust and safety and more. For analysis, we consider content and viewer $d$ well time (engagement duration) behaviors with images and videos on Snapchat Stories, one of the largest multimedia-driven social sharing services. To our knowledge, we are the first to model and analyze dwell time behaviors on such media. Specifically, our contributions include (a) individual modeling: we propose and evaluate the UM-DP, LM-DP and V-Dp parametric models to describe dwell times of unlooped/looped media and viewers which outperform alternatives, (b) aggregate modeling: we show how to flexibly summarize the respective joint distributions of multivariate parametrized fits across many samples using Vine Copulas in the analog UM-AM, Lм-AM and V-AM models, which enable inferences regarding aggregate behavioral patterns, and offer the ability to simulate real-looking engagement data (c) anomaly detection: we demonstrate our aggregate models can robustly detect anomalies present during training $(0.9+$ AUROC across most attack models), and also enable discovery of real dwell time anomalies.
\end{abstract}

\section{CCS CONCEPTS}

- Information systems $\rightarrow$ Web $\log$ analysis;

\section{KEYWORDS}

dwell time, modeling, multimedia, engagement

ACM Reference format:

Hemank Lamba and Neil Shah. 2019. Modeling Dwell Time Engagement on Visual Multimedia. In Proceedings of The 25th ACM SIGKDD Conference on Knowledge Discovery and Data Mining, Anchorage, AK, USA, August 4-8, 2019 (KDD '19), 10 pages.

https://doi.org/10.1145/3292500.3330973

\section{INTRODUCTION}

The recent years have brought about a tremendous increase in proliferation of visual multimedia content in the form of images and

\footnotetext{
${ }^{*}$ Work done while author was on internship at Snap Inc.

Permission to make digital or hard copies of all or part of this work for personal or classroom use is granted without fee provided that copies are not made or distributed for profit or commercial advantage and that copies bear this notice and the full citation on the first page. Copyrights for components of this work owned by others than ACM must be honored. Abstracting with credit is permitted. To copy otherwise, or republish, to post on servers or to redistribute to lists, requires prior specific permission and/or a fee. Request permissions from permissions@acm.org.

KDD '19, August 4-8, 2019, Anchorage, AK, USA

(C) 2019 Association for Computing Machinery.

ACM ISBN 978-1-4503-6201-6/19/08 _\$15.00

https://doi.org/10.1145/3292500.3330973
}

videos. Internet users watch 1 billion hours of YouTube video [2], share more than 95 million images and videos on Instagram [24], and spend an average of 30 minutes on Snapchat every day [23]. Dwell time, or engagement duration, is one of the key means of implicitly describing user interactions with content. In contrast to explicit features such as likes and follows, dwell time is not afflicted by low response rates and reporting bias. Content with high dwell time is considered more interesting and valuable to viewers, and indicate user attentiveness and satisfaction. Dwell time has thus been used as a central feature in content recommendation [14, 27, 28]. However, despite its value, prior work has left a considerable gap in modeling and analysis of dwell times on visual multimedia.

With the insight that dwell time can influence recommendations, numerous online marketplaces have spawned, offering customers ways to increase perceived engagement via paid inauthentic "views"; searches for "buy Youtube views" or "buy Instagram views" show numerous services offering bundles of 1 thousand views for as little as $10 \phi$. Such inauthentic engagement can disrupt recommendation algorithms, hurt advertiser profits, and increase user exposure to bad content. Despite this, prior work towards detecting abnormal viewer engagement using dwell times is nearly non-existent.

To bridge these gaps in behavior modeling and anomaly detection literature, we pose the following research questions:

- RQ1. Individual Modeling: How can we describe the dwell time distribution for a given content/viewer sample?

- RQ2. Aggregate Modeling: How can we jointly model dwell times across many content/viewer samples?

- RQ3. Anomaly Detection: Can such models help us detect dwell time engagement anomalies?

Dwell times have primarily been studied in the context of documents like webpages [14,26] and short articles [28]. To the best of our knowledge, ours is the first work that tackles the problem of modeling dwell times on visual multimedia. Our context poses a number of non-trivial challenges, including variety in varying content durations, media formats (looped and unlooped content) and behavioral diversity. Moreover, the sheer scale of engagement data is huge, necessitating scalable solutions for modeling. Our approach posits three core contributions, mirroring RQ1-RQ3:

- C1. Individual Modeling: We propose concise, interpretable parametric models which match empirical dwell time behaviors. Our proposed UM-DP (see Figure 1a), LM-DP and V-DP models for characterizing looped/unlooped media dwell times consistently outperform alternatives in terms of goodness-offit via 2-sample test and log-likelihood.

- C2. Aggregate Modeling: We propose aggregate models for looped/unlooped media (Lм-Aм/Uм-Aм) and viewer (V-Aм) dwell times, which utilize copulas to preserve multivariate dependency structures and model joint distributions of individual parameter fits (see Figure 1b). These models parametrically approximate original data with constant space, offer scalable inference, are temporally consistent and are also generative. 


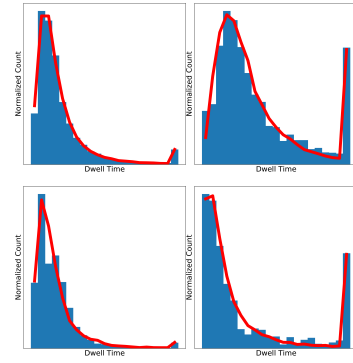

(a) Individual modeling

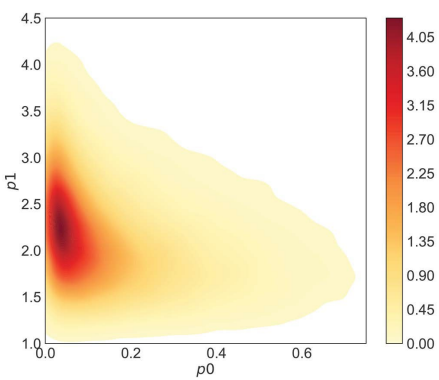

(b) Group modeling

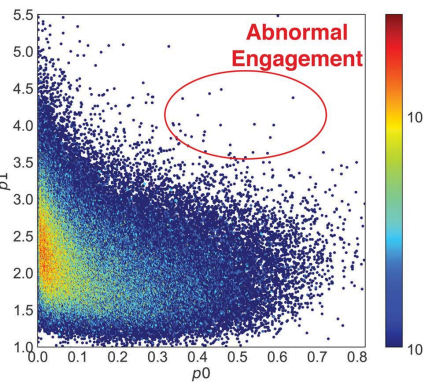

(c) Anomaly detection

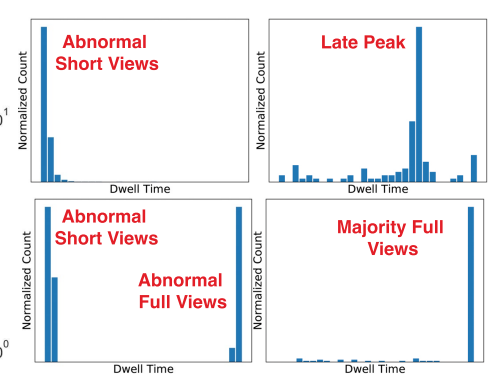

(d) Dwell time anomalies

Figure 1: Our work discusses (a) state-of-the-art parametric models for individual sample dwell times which closely mirror empirical data, (b) flexible copula modeling of aggregated multivariate parameter fits, (c) utilization of aggregate models for detecting dwell time engagement anomalies which (d) reflect abnormal behaviors radically inconsistent with most samples.

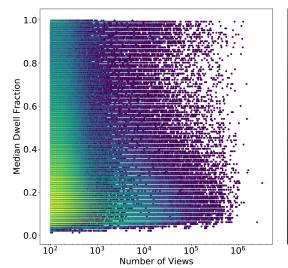

(a) Unlooped Media

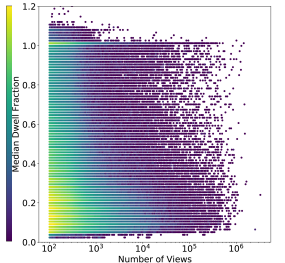

(b) Looped Media

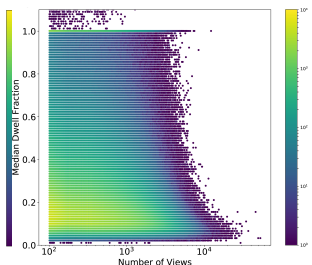

(c) Viewers

Figure 2: Median dwell time ratios vs. number of views on (a) unlooped and (b) looped media, and (c) viewers show outliers which exhibit excessively high dwell times compared to normal engagement patterns of similar view-count peers.

- C3. Anomaly Detection: We demonstrate that our aggregate models can be used to easily discover those with abnormal engagement (see Figures 1c/d). Experiments show our approach enables robust anomaly detection against simulated attacks (0.9+ AUROC in most experiments), and detects anomalous dwell time engagement behaviors on real data.

Though our work uses viewing data from Snapchat, we expect that given the diversity and scale of viewers and media settings that we consider, our findings should generalize on other visual multimedia platforms which support similar visual content types.

\section{RELATED WORK}

We discuss prior work in (a) temporal behavior modeling, and (b) detecting anomalous viewership.

Temporal behavior modeling. Prior work in dwell time modeling primarily focuses on recommendation and prediction of webpages and text documents. [28] explores interpreting dwell times as "pseudo-votes" for content recommendation of short-text documents; using a Log-normal distribution to model dwell times. [26] discusses using dwell times for re-ranking webpage results. [16] demonstrates that webpage features predicts the Weibull distribution modeled dwell times of webpage visits. [5,14] also discusses predicting dwell times on webpages and YouTube videos, respectively. Additionally, [3] proposed using gamma, weibull and exponential distribution to model dwell times. Several works focus on modeling temporal behaviors other than dwell times. [13] and [8] propose using the Log-logistic distribution to describe user interarrival times between search queries and forum comments. [25] uses a left-truncated Log-logistic model to describe human phone-call durations. Significant amount of work has been done in anomaly detection for

Table 1: Dataset summary

\begin{tabular}{ll}
\hline Unique media samples & 300 thousand \\
Images & 208 thousand \\
Videos & 92 thousand \\
Unlooped & 102 thousand \\
Looped & 198 thousand \\
\hline Unique viewers & 24 million \\
\hline Total views & 273 million \\
\hline
\end{tabular}

time-series [12], however our work is not concerned with modeling sequences, bur instead underlying distribution of dwell times.

Overall, unlike ours, none of the prior works (a) involve parametric dwell time modeling, (b) tackle general visual multimedia, and (c) model both users and content.

Detecting anomalous viewership. Prior literature in detecting anomalous viewership is sparse. [17] analyzed the fake view detection capacities of several video-sharing services including YouTube, DailyMotion, and Vimeo under synthetic attack models, demonstrating that all services were susceptible to simple attacks of fixed interarrival time views across IP addresses. [5] propose using user, IP and video entropies in a supervised model to detect abnormal engagement; however, their approach requires intensive manual labeling.[22] proposes using temporal view features in a livestreaming setting to detect distributional anomalies, but is non-parametric and does not expressly model dwell time behaviors, while being undefined for viewer and content anomalies.

Overall, unlike ours, none of the prior works (a) utilize implicit dwell time rather than explicit feedback, (b) tackle general visual multimedia, and (c) are unsupervised.

\section{DATA DESCRIPTION}

In this work, we study an industrial-scale media engagement dataset from Snapchat, one of the largest social multimedia-driven content sharing services. Snapchat enables users to share visual multimedia content to their "My Story," which can be optionally exposed to the entire userbase. Specifically, users can share ephemeral (purged in 24 hours) content (images or videos) with duration of up to 10 seconds, and adjust loop settings to unlooped (views automatically terminate upon completion) or looped (repeat indefinitely).

Our dataset consists of engagement associated with a large set of publicly posted "My Story" contents, and of the associated viewers for a long-enough time period sufficiently accounting for complete 


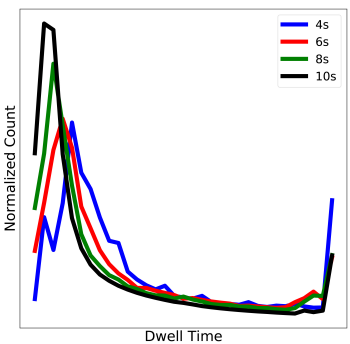

(a) Unlooped images

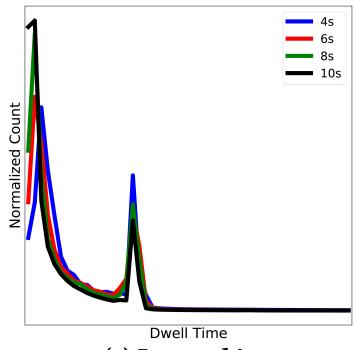

(c) Looped images

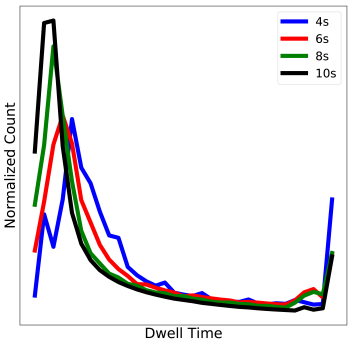

(b) Unlooped videos

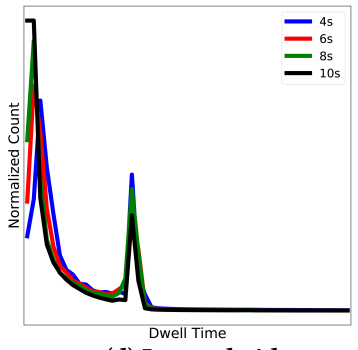

(d) Looped videos
Figure 3: Aggregated dwell time ratio statistics for varying media types and durations inform our modeling choices: treat images and videos similarly, and unlooped and looped content distinctly.

24-hour observation of engagement with all samples ${ }^{1}$. Table 1 details several key summary statistics of our dataset. All content samples and viewers have $100+$ associated views/data-points, enabling us to draw reasonably reliable inferences about engagement.

\section{INITIAL OBSERVATIONS}

Before delving into details, we conduct several exploratory analyses to motivate and direct our approach and give intuition for our subsequent modeling choices.

Firstly, we aim to understand dwell time behavior across the entire dataset, to determine patterns and anomalies in dwell times across different content and viewers. Since different content samples have varying durations, we normalize all dwell times with respect to these in order to compare them. Henceforth, when we mention "dwell time," we consider instead the dwell time fraction or ratio. Thus, dwell time ratios of views on unlooped media must lie in $(0,1]$, whereas dwell time ratios on looped content can lie on $(0, \infty)$.

Figure 2 shows quantized heatmaps of median dwell ratio of unlooped/looped media (2(a) and 2(b), respectively) and viewers (2c) versus view count, with brighter colors indicating logarithmically increasing density and darker colors denoting sparsity.Intuitively, sparsity increases towards the right of each plot due to skewed view count distributions, and towards the top of each plot, as few entities have high dwell ratios. Additionally, there are sparse entities in all plots which have very low dwell ratios. In all cases, we observe welldefined regions of high density.This suggests the following key observation, which motivates our modeling and anomaly detection goals.

Key Observation ((In)Consistencies in Visual Multimedia Dwell TIMEs). There exist patterns and anomalies in content and viewer dwell time engagement on visual multimedia.

\footnotetext{
${ }^{1}$ Due to privacy reasons, we obscure certain sensitive details (timeframes and certain axes values) while communicating our insights.
}

Next, we consider collective differences between unlooped and looped media, and their implications for dwell time modeling. Figure 3 shows the collective dwell ratios across our entire dataset, for unlooped images and videos in 3(a-b), and their looped counterparts in 3(c-d). The stark differences in distribution shape is apparent; unlooped dwell ratios are effectively censored at 1.0, where they achieve a second peak after a tapered drop. However, while looped dwell ratios exhibit a similar decay and noticeable peak at the first view "completion," (near mid-plot) they show a decreasing but nonzero probability afterwards due to differences in feasible view duration across the media types. This suggests the following:

Observation 1 (Looped/Unlooped Media Dwell Time DisparITY). Looped and unlooped media require characteristically different dwell time models, due to the differences in support over dwell time ratios of $(0,1]$ and $(0, \infty)$, respectively.

Lastly, we consider the effect of different media type (image and video) on dwell ratios. By comparing Figures 3(a)/(c) with 3(b)/(d), we can observe that images and videos actually admit very similar dwell times. Despite videos being intuitively "richer" than images, the plots mirror each other. Moreover, since we observe no significant differences in the "stickiness" across the collective media type splits, we hypothesize that a significant portion of users' decision to engage with content may actually occur before the user accesses the content, for example due to self-selection and preferences towards certain content. Our major takeaway regarding media types is thus

Observation 2 (Image/Video Dwell Time Parity). Dwell time similarities across image and video engagement suggest that they can be modeled characteristically similarly.

Given these observations, we next discuss our proposed parametric models for dwell time distributions of individual content samples and viewers; parametric models are appealing due to their conciseness and interpretability over nonparametric alternatives.

\section{INDIVIDUAL DWELL TIME MODELING}

How can we parametrically model the dwell time distributions of multimedia content and viewers? In this section, we first propose "dwell processes" to generatively model the multimedia content for both looped and unlooped media. Following this, we posit the same contributions for viewers. In both cases, we give the intuition behind our modeling approaches, discuss efficient parameter inference procedures and validate against alternatives using goodness-of-fit metrics.

\subsection{Multimedia Content Modeling}

5.1.1 Looped Content. We begin by discussing modeling of looped content. Views on such content are unbounded, and dwell time ratios can range from $(0, \infty)$. Given our earlier insights regarding longtailed dwell times from collective analysis in Figure 3, we consider several suitable distributions that may be able to model such shapes. In our preliminary analyses, we observed that the tails of many samples matched quite closely with Log-logistic distribution, defined as

Definition 5.1(Log-logistic(LL)Distribution). Let $T$ be a non-negative continuous random variable, such that $T \sim L L(\alpha, \beta)$. The PDF and CDF of $T$ are given by

$$
f_{L L}(t ; \alpha, \beta)=\frac{(\beta / \alpha)(t / \alpha)^{\beta-1}}{\left(1+(t / \alpha)^{\beta}\right)^{2}} \quad F_{L L}(t ; \alpha, \beta)=\frac{1}{1+(t / \alpha)^{-\beta}}
$$

where $t \in[0, \infty)$, and $\alpha$ (scale), $\beta>0$ (shape) are the parameters. 
Note that the $L L$ distribution admits the same support as our usecase for looped content, but does not do so for unlooped content. We propose using the original, unmodified $L L$ distribution as the core of our Lm-Dp (Looped Media Dwell Process), which can be written generatively as

Definition 5.2 (Looped Media Dwell Process $(L M-D P)$ ). Sample each dwell time ratio $t_{i} \sim L L(\alpha, \beta)$.

Use of $L L$ distribution over alternatives is justified for several reasons. $L L$ is widely used in survival modeling and has a hazard function implying that the longer a view has persisted, the longer it will continue to do so [1]. Also, it has demonstrated success in modeling other real-world temporal phenomena $[8,13]$ besides visual multimedia dwell times, and as we will show below, it outperforms other candidate distributions in this task.

Inference of LM-DP. Inference of $\alpha$ and $\beta$ cannot be computed in closed form. As a result, we infer parameters using the NelderMead simplex method [15], which maximizes likelihood via iterative approximations, while converging quickly and accurately.

Validation of LM-DP. We validate the model both qualitatively (visually) in terms of empirical versus simulated dwell time probabilities, and quantitatively via the Kolmogorov-Smirnov (KS) 2-sample test. In Figure 5, we illustrate the strong match in empirical dwell time distributions and our superimposed model fits across several looped media samples of varying exposure durations, viewer counts and dwell time behaviors. For brevity, we show results only on 6 users, but most others exhibited similar quality of fit. Observe that LM-Dp is able to well-approximate the peak and decay corresponding to view drop-offs reasonably well despite differences in distribution shapes across the samples, thus suggesting the appropriateness of our modeling choice.

To analyze the goodness-of-fit quantitatively, we perform KS tests comparing dwell times that were (a) empirically observed, with (b) those simulated by LM-DP using parameters inferred from MLE for each content sample. We compared LM-DP with four other alternative distributions which have previously been used for dwell time modeling in other contexts. These are CL-LN (Log-normal) [28], CL-IG (Inverse Gaussian) [11], CL-WB (Weibull) [16] and CL-G (Gamma) [14]. Figure 4(a) shows the sorted $p$-values reported across KS tests over samples reflecting the rejection probability for the null hypothesis $H_{0}$ that the empirical data and our simulated data are drawn from the same distribution. Assuming $H_{0}$ is true, the $p$-values should be uniformly distributed, manifesting as the $45^{\circ}$ line. We observe that our proposed LM-DP using $L L$ performs the best, with the CL-LN model the next closest, CL-IG/CL-WB and CL-G demonstrating significantly worse performance. Figure 4(b) further shows the percentage of samples that were fitted "successfully" $(\mathrm{KS} p<.05)$ given their view counts. Again, we observe that LM-Dp outperforms competitors, modeling the vast majority of samples successfully (over $90 \%$ for

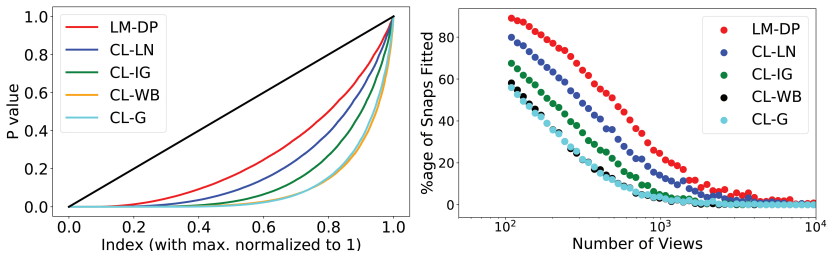

Figure 4: LM-DP outperforms alternatives:(a) sorted $p$-values from KS tests; the closer a model curve to the $45^{\circ}$ line, better the fit. (b) \%age of samples where model fits were $\operatorname{successful}(p<.05)$.
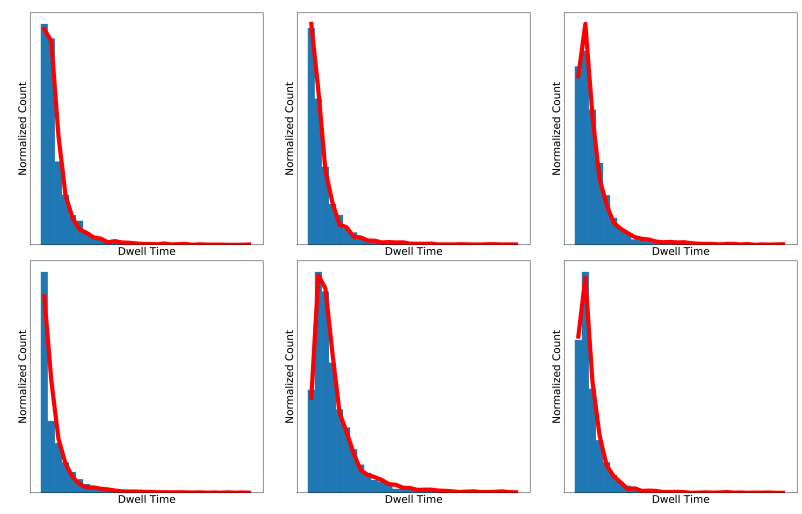

Figure 5: Proposed Lm-DP (red) visually matches empirical dwell times (blue) across several looped media samples of varying patterns.

Table 2: \% of instances where proposed models outperforms alternatives (higher is better, $>50 \%$ implies superior performance).

\begin{tabular}{ccccc}
\hline LM-DP & CL-LN & CL-IG & CL-WB & CL-G \\
\hline NLL & $54.5 \%$ & $82.4 \%$ & $94.6 \%$ & 93.7 \\
KS & $78.9 \%$ & $86.2 \%$ & $84.7 \%$ & 86.7 \\
\hline UM-DP & CU-LN & CU-IG & CU-WB & CU-G \\
\hline NLL & $53.6 \%$ & $78.2 \%$ & $84.1 \%$ & 85.5 \\
KS & $73.2 \%$ & $86.7 \%$ & $88.9 \%$ & 90.2 \\
\hline V-DP & CV-LL & CV-IG & CV-WB & CV-G \\
\hline NLL & $93.6 \%$ & $82.6 \%$ & $99.1 \%$ & $99.9 \%$ \\
K-S & $52.8 \%$ & $54.1 \%$ & $81.1 \%$ & $84.1 \%$ \\
\hline
\end{tabular}

samples with $\approx 100$ views). Note that since KS tests and $p$-values are highly sensitive given large sample sizes (i.e. $H_{0}$ would be rejected even for minute differences between empirical and simulated data), the percent of successful fits decreases in all cases with high view count; however, given the skewed distribution of view counts, high view count cases constitute only a small fraction of the population. Table 2(LM-Dp) further demonstrates the aggregated percentage of samples for which LM-DP outperforms the alternatives, according to both KS test $p$-values and negative log-likelihood (NLL) of the fitted models; note that these differences are significant and persistent across hundreds of thousands of samples. Additionally, since all the models have same number of parameters, model complexity metrics are proportional to NLL and hence we do not explicitly mention them.

5.1.2 Unlooped Content. Unlike for looped media, unlooped views can have a maximum dwell ratio of 1.0 given viewing constraints (discussed in Observation 1). We observe that no typical continuous value distributions are able to handle this constraint on support natively. Therefore, we propose our UM-DP (Unlooped Media Dwell Process) which significantly augments the LM-Dp to handle this constraint. The model can be written generatively as

Definition 5.3 (Unlooped Media Dwell Process $\left.\left(U_{M}-D P\right)\right)$. Sample each dwell time ratio $t_{i}$ as

(1) $c_{i} \sim \operatorname{Bernoulli}(\theta)$

(2) $t_{i} \sim\left\{\begin{array}{lll}\delta_{1}(\cdot) & \text { if } c_{i}=1 \\ T L L(\alpha, \beta) & \text { if } c_{i}=0 & \text { [complete view] }\end{array}\right.$ where $f_{T L L}(t ; \alpha, \beta)=f_{L L}(t ; \alpha, \beta) / Z$ is the PDF of right-truncated $L L$ distribution on $t_{i} \in(0,1), Z=F_{L L}(t=1 ; \alpha, \beta)-F_{L L}(t=0 ; \alpha, \beta)$ for normalization, and $\delta_{1}(\cdot)$ denotes a point mass at 1.0. 

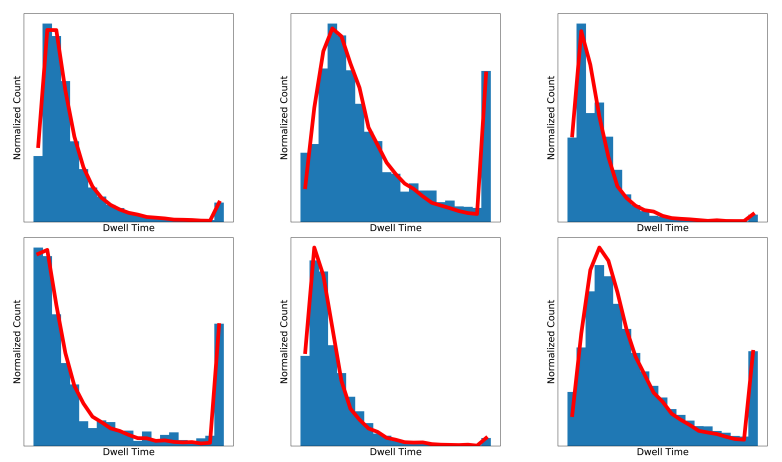

Figure 6: Our proposed UM-DP (red) visually matches empirical dwell time probabilities (blue) across unlooped media samples with varying viewing patterns.

The main idea behind $\mathrm{UM}_{M}-\mathrm{DP}$ is that it considers separately the cases where (a) viewers make a preemptive choice to consume the complete media content (due to friendship, self-selection, etc.), and (b) viewers are less invested and drop off when they lose interest. Intuitively, this reflects a dichotomous choice in media consumption: sometimes, we "exploit" the media which we highly suspect to be interesting given factors like interest in the poster, subscriptions, fascination with a content thumbnail, etc., and other times we "explore" other content whom we give attention to in a fickle way. We note that UM-Dp bears resemblance to hurdle models, which are often used to model over-inflation of 0 s in ecologicaldata[21]; such models pose a "hurdle" via a Bernoulli probability, which when overcome allows a non-zero sample to be generated from an auxiliary process. Our UM-Dp places such a hurdle of probability $\theta$ on $P(t=1.0)$ to model complete views, and with probability $1-\theta$ we sample from the auxiliary $T L L$ distribution such that $t<1.0$ for truncated views. Inference of UM-DP. We infer parameters for UM-DP by maximizing the log-likelihood. The overall log-likelihood is given by

$$
\ell(\theta, \alpha, \beta)=\sum \theta \log P\left(t_{i}=1.0\right)+(1-\theta) \log f_{T L L}\left(t_{i} ; \alpha, \beta\right)
$$

We can infer $\theta$ by maximum likelihood by taking the proportion of empirically observed complete views, i.e. $\hat{\theta}=\sum 1\left(t_{i}=1.0\right) / n$ over $n$ total views. After filtering the complete views, we can estimate the $\alpha, \beta$ parameters for $T L L$ on the truncated views by maximizing likelihood heuristically as in the LM-Dp case.

Validation of UM-DP. Again, we validate the model both qualitatively and quantitatively. Figure 6 illustrates parity between empirical data and superimposed model fits across unlooped media samples of varying durations, viewer counts and dwell time behaviors. We observe that our proposed UM-DP is able to well-approximate both the completed views (far right), and maintains good performance in modeling the peak/decay corresponding to viewer drop-off despite differences in distribution shapes.

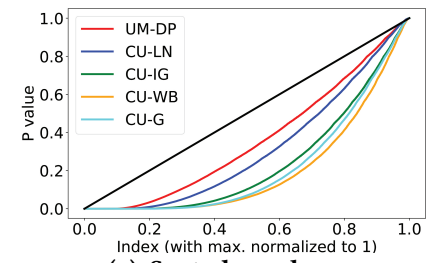

(a) Sorted $p$-values

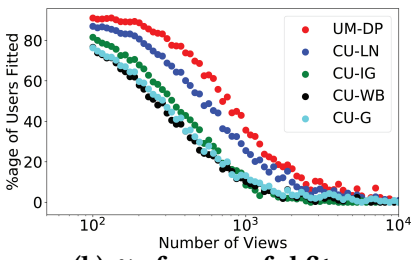

(b) $\%$ of successful fits
Figure 7: UM-DP outperforms alternatives:(a) sorted $p$-values from KS tests; the closer a model curve to the $45^{\circ}$ line, better the fit. (b) \%age of samples where model fits were $\operatorname{successful}(p<.05)$.
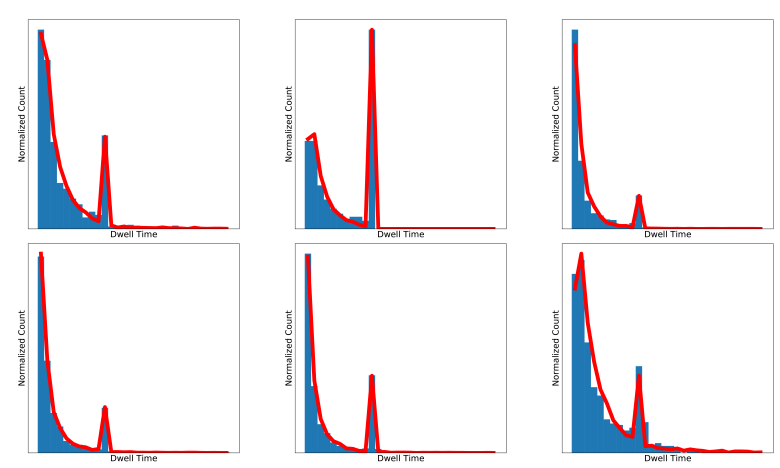

Figure 8: Our proposed V-Dp (red) visually matches empirical dwell times (blue) across several looped media samples with varying viewing patterns.

Quantitatively, we again used KS tests and NLL to compare performance of UM-DP with alternatives: CU-LN (Log-normal), CU-IG (Inverse Gaussian), CU-WB (Weibull) and CU-G (Gamma). Technically, we used the truncated variants of these models in the same context proposed in our UM-Dp formulation. Figure 7 shows the sorted $p$-values across samples in (a) and percentage of samples correctly fit against view count in (b); again, we observe that the proposed $\mathrm{UM}_{\mathrm{M}} \mathrm{DP}$ fits the majority of samples well (around $90 \%$ with $\approx 100$ views) outperforms the other models, with the CU-LN model the next closest, and CU-IG/CU-WB demonstrating significantly worse performance. Table 2(UM-Dp) further shows that $U_{M}$-Dp outperforms the alternatives over aggregated percentage of samples better fit by both KS tests and log-likelihood comparisons across fitted models.

\subsection{Viewers}

Modeling viewers has a distinct set of challenges. Most notably, we must model viewers across time that they spend on looped and unlooped media both. Given the differences in support over dwell time ratios over the two, this is non-trivial. Moreover, we must account for differences in inherent propensities of viewers to watch unlooped and looped media. The alternatives to accounting for these complexities in a single joint model are undesirable, as they would result in having individualized models for each user across multiple content types and exposure durations, greatly increasing model complexity and requiring many more samples for inference.

To overcome these challenges, we propose V-Dp (Viewer Dwell Process), which aims to unify the modeling of these heterogeneous phenomena. At the core of V-DP is the Log-normal distribution, which we observed closely matched the tails of many viewers' dwell time ratios. The Log-normal distribution is defined as

Definition 5.4 (Log-normal Distribution (LN)). Let $T$ be a nonnegative continuous random variable, such that $T \sim L N(\mu, \sigma)$. The $\mathrm{PDF}$ and $\mathrm{CDF}$ of $T$ are given by:

$$
f_{L N}(t ; \mu, \sigma)=\frac{1}{t \sigma \sqrt{2 \pi}} e^{-\frac{(\log t-\mu)^{2}}{2 \sigma^{2}}} \quad F_{L N}(t ; \mu, \sigma)=\Phi\left(\frac{\log t-\mu}{\sigma}\right)
$$
where $t \in(0, \infty), \mu \in(-\infty, \infty)$ and $\sigma>0$ are the mean and standard deviation of $\log T$, and $\Phi$ indicates the standard normal CDF.

Like $L L$, the $L N$ distribution is also commonly used in survival analysis [7]. Both distributions have very similar shapes; however, $L L$ typically has heavier tails. Intuitively, this disparity in distributions between content-centric and viewer-centric modeling makes sense as viewers have more associated "outgoing" views than contents 
have "incoming" ones, and proportionally more of those views tend to be short. This would explain why viewer dwell time ratios exhibit more probability in the head of the distribution with lighter tails, making $L N$ a more suitable option for the viewer modeling task than $L L$. Given this, we propose the V-Dp as follows.

Definition 5.5 (V-DP). Sample each dwell time ratio $t_{i}$ as

(1) $l_{i} \sim$ Bernoulli $(\psi)$

(2) $c_{i} \sim \operatorname{Bernoulli}(\theta)$

(3) $t_{i} \sim\left\{\begin{array}{lll}L N\left(t ; \mu_{L}, \sigma_{L}\right) & \text { if } l_{i}=0 & \text { [LM view] } \\ \delta_{1}(\cdot) & \text { if } l_{i}=1, c_{i}=1 & \text { [UM comp. view] } \\ T L N\left(\mu_{U}, \sigma_{U}\right) & \text { if } l_{i}=1, c_{i}=0 & \text { [UM trunc. view] }\end{array}\right.$ where $f_{T L N}(t ; \mu, \sigma)=f_{L N}(t ; \mu, \sigma) / Z$ is the PDF of right-truncated $L N$ distribution on $t_{i} \in(0,1), Z=F_{L L}(t=1 ; \alpha, \beta)-F_{L L}(t=0 ; \alpha, \beta)$ for normalization, and $\delta_{1}(\cdot)$ denotes a point mass at 1.0.

Our proposed V-Dp is a mixture of viewing processes between both looped and unlooped content. The unlooped content has a max dwell time ratio of 1.0, and thus we sample views to this content in a manner similar to UM-DP, with the exception of using TLN distribution. Looped content has views with unbounded dwell time ratios, and thus we sample these views in a manner similar to LM-DP, but using $L N$ distribution. The mixture proportions are determined by a parameter trading off propensity for looped versus unlooped media. Note that here, we model views to content with different exposure durations in the same, dwell time ratio model. Technically, though we describe the unlooped and looped views using a single $L N$ variant each, we are actually observing the convolution of the underlying varying duration distributions.

Inference of V-DP. We aim to maximize the log-likelihood in inferring parameters for V-DP. The log-likelihood of is given by

$$
\begin{aligned}
\ell\left(\psi, \theta, \mu_{U}, \sigma_{U}, \mu_{L}, \sigma_{L} \mid t\right)= & \sum \psi\left[\theta \log P\left(t_{i}=1.0\right)\right. \\
& \left.+(1-\theta) \log f_{T L N}\left(t_{i} ; \mu_{U}, \sigma_{U}\right)\right] \\
& +(1-\psi) \log f_{L N}\left(t_{i} ; \mu_{L}, \sigma_{L}\right)
\end{aligned}
$$

Consider $n$ as the total number of views, and $n_{U}$ and $n_{L}$ as number of views on unlooped and looped content (such that $n_{U}+n_{L}=n$ ). Then, we have $\hat{\psi}=n_{U} / n$, and similarly if we consider the number of complete views on unlooped snaps as $n_{U}^{C}$, then $\hat{\theta}=n_{U}^{C} / n_{U}$. To infer parameters $\mu_{L}, \sigma_{L}$ for looped media views, we can use closed form estimators. To infer the $L N$ parameters $\mu_{U}, \sigma_{U}$ for unlooped snaps, we maximize the TLN log-likelihood using Nelder-Mead.

Validation of V-DP. We validate V-Dp both qualitatively and quantitatively. Figure 8 shows several example fits of V-Dp on sample viewers; observe that our formulation allows a flexible fitting of various, complex distributional shapes which represent engagement with highly heterogeneous content using few parameters. Moreover, we compare V-Dp with other candidate models, which as in UM-DP and LM-DP, differ from V-DP in the central parametric distribution used. The candidate models CV-LL (Log-Logistic), CV-IG (Inverse Gaussian), CV-WB (Weibull) and CV-G (Gamma), differing in replacement of $L N$ distribution to respectively mentioned ones.

Quantitatively, we evaluate V-DP's goodness-of-fit by using KS tests and NLL. We plot the sorted $p$-values of V-DP and alternatives in Figure 9(a), demonstrating that V-Dp's $p$-value curve is closest to the ideal and fits better than alternatives, with CV-LL coming in at a close second. Figure 9(b) shows the percentage of viewers that are successfully fit with V-Dp; here too, we observe that V-Dp fits for majority of the viewers (over $95 \%$ with $\approx 100$ views) with CV-LL performing roughly on par at lower view counts, but trailing behind (a) Sorted $p$-values

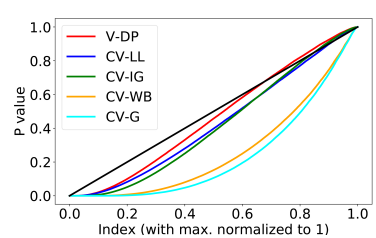

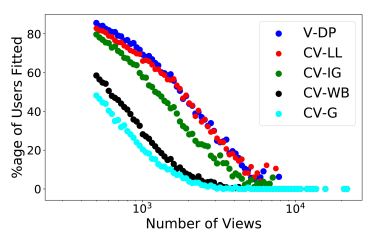

(b) \% of successful fits
Figure 9: V-Dp outperforms alternatives:(a) sorted $p$-values from KS tests; the closer a model curve to the $45^{\circ}$ line, better the fit. (b) \%age of samples where model fits were successful $(p<.05)$.

as view count increases. Again, decrease in fit performance at high view count is encountered by all models due to KS test sensitivity with large sample sizes. Table 2(V-Dp) lists the aggregate percentage of cases where V-Dp performs better than other candidate models in both KS $p$-values and NLL; NLL suggests significantly better fit performance using V-Dp over the competitors.

\section{AGGREGATE DWELL TIME MODELING}

Given parametric individual fits for each individual content or viewer sample, how can we identify patterns, normative behaviors and anomalies in dwell times of many content or viewer samples, respectively? How common is it to watch over $80 \%$ of an image or video? How common is it for a viewer's dwell times to be narrowly distributed around 5\% and so on? To answer the above questions, we need to model the parameters in aggregate, across many samples. However, modeling the joint distribution of multivariate data is in general not trivial, posing challenges in dependency estimation, inference and curse of dimensionality. In this work, we propose to flexibly model joint distributions of parameters across many content and viewer samples respectively, using a powerful statistical tool known as a copula [20]. Copulas allow for scalable, parametric, approximate inference of multivariate distributions. This second level of parametricity in our modeling is advantageous, as it helps us better interpret inter-parameter dependency estimation, enables quick normality scoring and likelihood estimation, and moreover is generative, letting us actually simulate high-quality, realistic dwell time data.

\subsection{Copula Modeling}

6.1.1 Bivariate Modeling. Copulas are statistical tools, that explicitly model the dependency structure between given univariate marginals to estimate bivariate joint distributions. Copulas have been extensively used in finance [4], healthcare [18] and hydrology research [9]. We can define a bivariate copula as follows:

Definition 6.1 (Bivariate Copula). A bivariate copula $C$ is a dependency function, defined as $C:[0,1]^{2} \rightarrow[0,1]$. Given two random variables $U$ and $V$ and their marginal CDFs $F_{U}$ and $F_{V}$, a copula $C\left(F_{U}(u), F_{V}(v)\right)$ models the joint $\mathrm{CDF}$, admitting a joint PDF of

$$
f_{U, V}(u, v)=f_{U}(u) \cdot f_{V}(v) \cdot c\left(F_{U}(u), F_{V}(v)\right)
$$

where $c$ and $f$ denote copula and marginal densities.

Technically, copulas are defined on uniform marginal CDFs. We can transform any random variable $Y$ to uniformity by using probability integral transform (PIT) or vice-versa (inverse transform sampling). Various parametric forms of copula exist and can be used to capture different dependencies (positive, negative, independent) between different types of random variables. While bivariate copulas have demonstrated great empirical success in capturing dependencies via a variety of parametric forms, the number of generalized 
multivariate parametric copulas (for $>2$ variables) are highly limited and inflexible in preserving pairwise dependencies, resulting in poor estimation. Given that some of our proposed models are multivariate, we seek a better option: to model multivariate dependencies parametrically while also allowing for flexible pairwise dependency modeling in high dimension, we propose the use of Vine copulas.

6.1.2 Multivariate Modeling. Vine copulas leverage the flexibility of parametric bivariate copulas to preserve bivariate statistical dependencies in higher-dimensional joint distributions. The dependency structure is modeled by the composition of (a) a set of bivariate copula families, (b) the associated copula dependency parameters, and (c) a nested tree structure to model the decomposition of joint distribution into the bivariate copula and marginal densities, as follows [6]

Definition 6.2 (Vine copula). A vine copula on $n$ random variables $X_{1} \ldots X_{n}$ has a joint PDF defined by

$$
f_{X_{1} \ldots X_{n}}\left(x_{1} \ldots x_{n}\right)=\prod \prod c_{i, i+j \mid i+1, \ldots, i+j-1} \cdot \prod f_{k}\left(x_{k}\right)
$$

where $c$ and $f$ denote associated copula and marginal densities.

Different tree structures have been proposed to model these dependencies ; in this work, we use canonical vines ( $C$-vines). $C$-vines decompose marginals and bivariate copula densities such that every tree has a one-to-many structure:

Definition $6.3\left(C\right.$-vine). A set of linked trees $\mathcal{V}=\left(T_{1}, T_{2}, T_{n-1}\right)$ is a $C$-vine on $n$ elements if

(1) $T_{1}$ is a tree with nodes $N_{1}=1, \ldots, n$ and a set of edges $E_{1}$ between a selected node $a \in T_{1}$ and all other nodes $b \in T_{1}$.

(2) For $i=2, \ldots, n-1, T_{i}$ is a tree with $E_{i-1}$ nodes and edge set $E_{i}$ such that a single node in $T_{i}$ is connected to all other nodes in $T_{i}$, and no other edges exist.

Inference. To select the appropriate $C$-vine structure, we use the procedure as mentioned in [6]; specifically the node with maximum absolute Kendall's $\tau$-correlation to other nodes is selected as central node for each level tree. Given the structure, we maximize loglikelihood to infer bivariate copulas and the associated parameters.

\subsection{Multimedia Content}

Below, we discuss how we conducted modeled aggregate modeling for looped and unlooped media.

6.2.1 Looped Content. Since our LM-Dp produces only 2 parameters for each content sample, a bivariate copula suffices to model the two-parameter dependency. To do this, we used LM-Dp to to fit parameters for all looped media samples, and subsequently applied the PIT using the empirical CDFs for both $\alpha$ and $\beta$ describing the dwell ratio scale and shape. We then selected the bivariate copula (shown in Figure 10(a)) which best maximizes the log-likelihood across a variety of parametric forms discussed in [19], and inferred parameters using $30 \%$ of the samples; we call this model LM-AM.

6.2.2 Unlooped Content. We obtained 3 parameters from UM-DP, $\theta, \alpha, \beta$ which describe view completion rate and truncated view dwell time ratio scale and shape. Given the multivariate setting, we inferred parameters for a Vine copula (shown in Figure 10(b)), training on $30 \%$ of the samples as in the looped case. We call this model UM-Am.

\subsection{Viewers}

In modeling individual viewer dwell ratios, our V-Dp produced 6 parameters for each viewer: $\psi, \theta, \mu_{L}, \sigma_{L}, \mu_{U}, \sigma_{U}$, denoting propensity

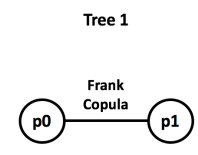

(a) Lм-Aм (Looped Media)

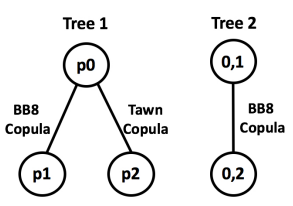

(b) UM-Aм (Unlooped Media)
Figure 10: Bivariate and $C$-vine copula structures can model joint densities parametrically. (a) and (b) show our LM-АM and Uм-АM dependency structures, respectively.

Table 3: Pearson correlation coefficients between parameters in original and simulated data.

\begin{tabular}{cccc}
\hline Correlations & $(\mathrm{p} 0, \mathrm{p} 1)$ & $(\mathrm{p} 1, \mathrm{p} 2)$ & $(\mathrm{p} 0, \mathrm{p} 2)$ \\
\hline Original & -0.32 & 0.08 & 0.43 \\
Simulated & -0.31 & 0.10 & 0.41 \\
\hline
\end{tabular}

to view unlooped media, propensity to complete unlooped views, and mean and standard deviation of the log dwell ratios for looped and truncated unlooped views, respectively. Using a sample of $100 \mathrm{~K}$ instances, we estimated and fitted a $C$-vine. We call this model V-AM.

\subsection{Validation}

To evaluate the performance of $C$-vine modeling in our usecase, we consider the following aspects:

- Q1. Dependency preservation: How well does $C$-vine approximate the original data dependencies?

- Q2. Training size: How is $C$-vine modeling performance influenced by training size?

- Q3. Temporal consistency: How robust is $C$-vine modeling for similar data from two different time-frames?

Given space constraints, we show experimental results only on $\mathrm{UM}-\mathrm{Am}$, noting that those for LM-AM and V-AM are similar.

6.4.1 Dependency preservation. Here, we determine if dependency structure in original data is well approximated by the $C$-vine model. To this end, we compare generated random samples from the simulated data on $[0,1]^{n}$ ( $n=3$ for UM-AM, used here) to the PITrepresentation of training samples. We report the pairwise Pearson correlations in Table 3, and show heatmaps of the pairwise dependencies in Figure 11. We observe that correlations between all parameter pairs and density estimates are closely approximated.

6.4.2 Training size. We also study the effects of training size on $C$-vine modeling performance. We experimented by training the $C$ vine model using random samples of varying sizes from the entire set, and sampling instances from the fitted models. To comparing samples from multivariate distributions, we use kernel-based Maximum Mean Discrepancy (MMD) test as proposed in [10] (the KS test is only suitable for univariate samples), to test the null hypothesis $H_{0}$ : simulated samples and original data samples are from same distribution. We present the MMD test statistic for data simulated using models with various training sizes in Table 4; results show that we are not able to reject $H_{0}$ in any case. Even when using only $10 \%$ training data, we observe that the $C$-vine model closely approximates the original data distribution. Notice that the MMD statistic decreases as training size increases, showing closer approximation towards the original data.

6.4.3 Temporal consistency. We next aim to validate that aggregate models produced from $C$-vine are temporally consistent, in that they closely match across data taken from different time periods 
(we expect the underlying behavior does not shift significantly). We fit another $C$-vine model using dwell time engagement data from a different month than the data discussed here. We then compared the simulated data from both $C$-vine models and evaluated similarity between the two. To this end, we perform an MMD test between the two samples, obtaining a test statistic of 0.032 , which does not let us reject $H_{0}$, and thus we can say they are drawn from same distribution. We observe this visually in Figure 12, where samples generated from both $C$-vines produce similar dependency structures for each pair.

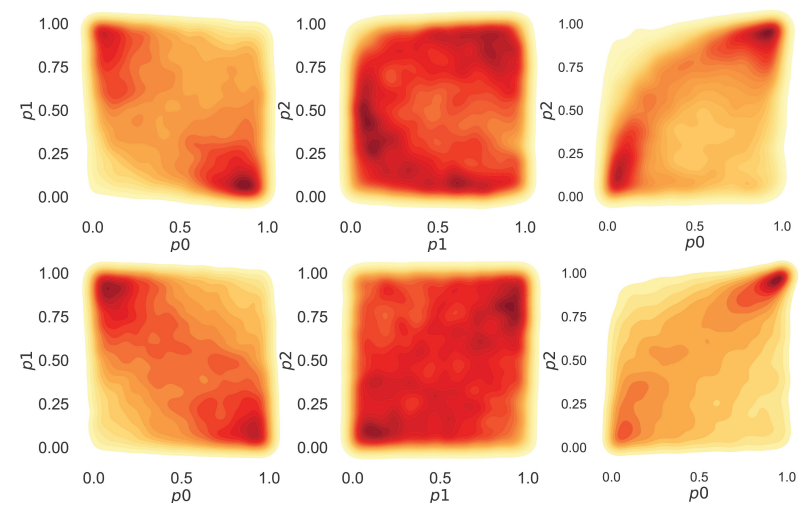

Figure 11: Aggregate $C$-vine models closely approximate real data. Pairwise dependency heatmaps between original data (top) and simulated data (bottom) are visually close.
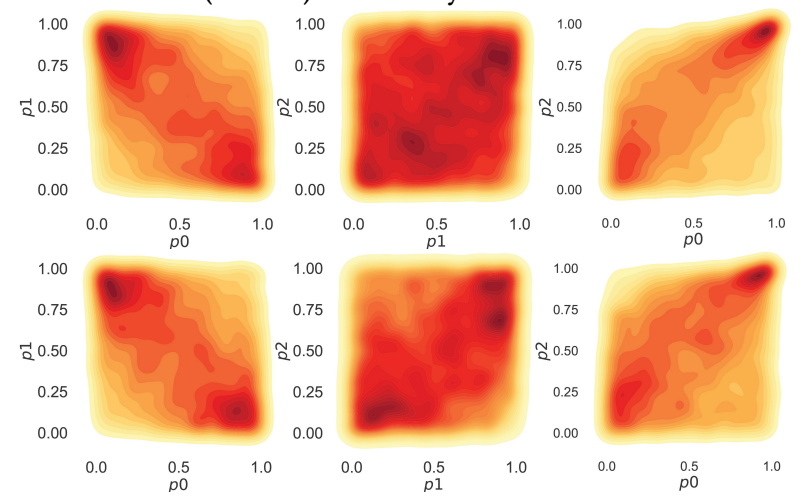

Figure 12: $C$-vine models are robust and consistent over time. Pairwise dependency heatmaps between simulated data from aggregate models trained on two different months (top and bottom) are visually close.

\section{ANOMALY DETECTION}

In the previous section, we introduced parametric copula-based models for aggregate content and viewer modeling, demonstrating success in modeling the vast majority of samples while preserving complex interactions between parameters. A natural line of evaluation is determining effectiveness of such models in detecting anomalous engagement samples(i.e. samples which have extremely low-likelihood according to the aggregate models); thus, we pose the following questions.

Table 4: MMD test statistics between original data and modelsimulated data (lower is better).

\begin{tabular}{cccccc}
\hline Training Size & $10 \%$ & $20 \%$ & $30 \%$ & $40 \%$ & $50 \%$ \\
\hline MMD-Statistic & 0.037 & 0.034 & 0.0334 & 0.333 & 0.30 \\
Reject $H_{0}$ & No & No & No & No & No \\
\hline
\end{tabular}

- Q1. Robustness to contamination: Can our aggregate models robustly detect anomalies under contamination?

- Q2. Qualitative efficacy: Do our aggregate models detect real engagement anomalies on real data?

\subsection{Robustness to contamination}

We first study the performance of our aggregate model in detecting anomalies present in the training data, known as contamination. This scenario is possible in unsupervised models, like ours, as anomalous samples are not labeled and are also involved in individual and aggregate modeling steps. Ideally, our models should be able to detect anomalies in training data with high precision. To evaluate performance in such settings, we analyze our model's ability to successfully detect simulated attacks, by means of injecting artificial, anomalous samples in the original data. We present results for only UM-Am given space constraints, but results for LM-Am and V-Am were similar.

We consider 4 different contamination models (shown below) in which anomalies are generated (a) according to different attack models, and (b) constituting varying contamination ratios. Model 1 (Complete Views): Anomalies have all fully complete views: dwell time ratios of 1.0, Model 2 (Long Views): Anomalies have overly long views: dwell time ratios sampled uniformly between $0.8-1.0$, Model 3 (Short Views): Anomalies have overly short views: dwell time ratios Gamma-distributed such that most dwell time ratios $<0.2$, and Model 4 (Uniform Views): Anomalies have randomlength views: dwell time ratios sampled uniformly on $0.0-1.0$. Also, we consider varying contamination ratios of $1 \%, 2 \%$ and $5 \%$ anomalies in the training data. We evaluate detection capacity via AUROC, which is reliable in imbalanced class settings like ours. Results are shown in Table 5, and indicate extremely high detection performance. We observe an AUC of $0.9+$ across most scenarios, noting that higher contamination results intuitively result in lower AUC due to increased model corruption.

\subsection{Effectiveness on real data}

Next, we aim to evaluate whether our models can actually detect anomalous dwell time engagement in real data. To this end, we selected the 1000 most normal and anomalous samples according to log-likelihood, for each looped/unlooped content sample and viewer under Lм-Ам, Uм-Ам and V-Am respectively, and compared the empirical CDFs of mean dwell times across these entities. Intuitively, if our aggregate models were not detecting anomalous engagement, the empirical CDFs would closely match. However, as Figure 13 shows, the curves are significantly different for normal and anomalous samples identified by each model; note that the $x$-axis is in log-scale, making the observed differences more significant. We observe clear differences throughout the range of the CDF, and moreover discover the biggest differences near the extremities, suggesting our model does detects engagement anomalies. At the lower extremity of dwell time ratios, we observe that the lowest anomalous samples dwell times were $3-5 \times$ smaller than those of their normal counterparts. Likewise, at the upper extremity, the highest anomalous sample dwell times were $2-4 \times$ larger.

Manual inspection of several observed anomalous dwell time behaviors indicated significant abnormalities: (1) One anomalous viewer had over 5000 views/day, with mean dwell ratio $<0.03$, and was adding more than 200 friends/day from an already staggering 3900, (2) several anomalous looped media samples with over 500 views had mean dwell ratio of $10-15 \times$ the duration, and (3) several unlooped media samples with $100-300$ views had mean dwell ratios 
Table 5: Anomaly detection performance (AUROC) under various anomaly contamination \%ages (higher is better).

\begin{tabular}{cccc}
\hline Attack Model & $1 \%$ & $2 \%$ & $5 \%$ \\
\hline Model 1 (Full Views) & 0.99 & 0.98 & 0.96 \\
Model 2 (Long Views) & 1.0 & 0.99 & 0.99 \\
Model 3 (Short Views) & 1.0 & 0.99 & 0.98 \\
Model 4 (Uniform Views) & 0.94 & 0.92 & 0.84 \\
\hline
\end{tabular}

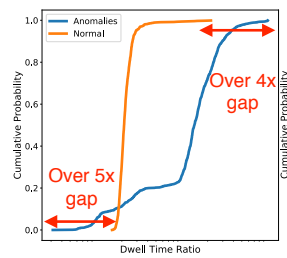

(a) Looped content

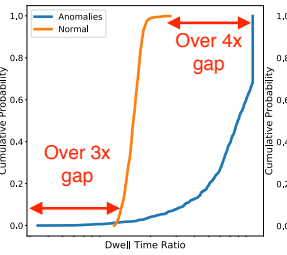

(b) Unlooped content

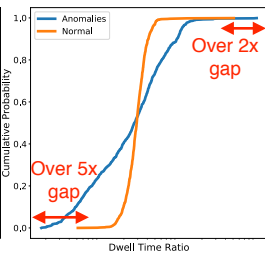

(c) Viewers
Figure 13: Our aggregate models detect real dwell time anomalies. The subplots show huge disparities in the mean dwell time ratio distributions between anomalous and normal (a) unlooped media, (b) looped media and (c) viewer samples.
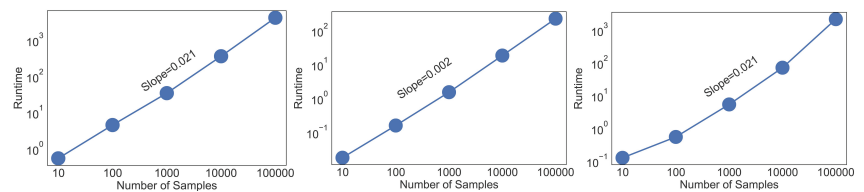

(a) Individual fitting (b) PIT pre-processing (c) Copula inference

Figure 14: Our model inference is scalable: (a-c) show that individual fitting, copula preprocessing via integral transform, and copula inference are all near-linear in sample size.

of over 0.9 ; one sample with over 1000 views had a ratio of just 0.03 . Figure 1(d) shows several examples of unlooped content anomalies discovered by Uм-Ам (others excluded for brevity). These anomalies could correspond to fake engagement, or possibly offensive or polarizing media. Overall, results demonstrate that our approach does empirically detect real-world anomalies across aggregate models, and could be additionally correlated with other features to discern abusive behaviors of various types.

\section{SCALABILITY}

We briefly discuss scalability in terms of both individual dwell process fitting and aggregate copula modeling. The major runtime cost in the former case is log-likelihood maximization for fitting parameters of the relevant dwell process.Figure 14(a) shows that this procedure exhibits empirically linear runtime in the number of training samples. The runtime costs in the latter case are incurred in conducting the PIT on original data samples for copula pre-processing, and selecting ideal copula structure and parameters. Figures 14(b) and 14(c) show that these steps admit linear and near-linear runtime respectively. Results are shown on $\mathrm{UM}_{M}-\mathrm{Dp} / \mathrm{UM}-\mathrm{Am}$.

\section{CONCLUSION}

In this work, we provide the first comprehensive analysis of modeling dwell time engagement on visual multimedia content. Studying such content is valuable, as its consumption constitutes a significant portion of daily online activity, and has valuable applications in behavior modeling and anomaly detection. We first discuss challenges and considerations in the modeling task, including content heterogeneity and behavioral diversity. Our first contribution constitutes the LMDp, UM-DP and V-Dp generative dwell time processes and inference procedures, which enable individual modeling of content-centric and viewer-centric dwell time engagement. We show that these models match empirical data visually and quantitatively according to KS tests and outperform alternatives in both log-likelihood and KS tests. Our next contribution posits the analog LM-AM, UM-AM and V-DP, which enable aggregate modeling of joint distributions across individual fits using parametric bi/multivariate copulas. We demonstrate the flexibility of such models in capturing high dimensional dependencies with limited training data, show that they closely match original data both visually and quantitatively according to MMD tests, and are temporally consistent. Our last contribution includes ramifications of our proposed models for anomaly detection, in both robustness to contamination (0.9+ AUROC in most experiments) and qualitative evidence in terms of anomalies detected on real engagement data.

\section{REFERENCES}

[1] Steve Bennett. 1983. Log-logistic regression models for survival data. Applied Statistics (1983).

[2] YouTube Offical Blog. 2017. You know whatś cool? A billion hours. https://goo.gl/zPNNT7. (2017).

[3] Alexey Borisov, Ilya Markov, Maarten de Rijke, and Pavel Serdyukov. 2016. A context-aware time model for web search. In SIGIR.

[4] Eric Bouyé, Valdo Durrleman, Ashkan Nikeghbali, Gaël Riboulet, and Thierry Roncalli. 2000. Copulas for finance-a reading guide and some applications. (2000).

[5] Liang Chen, Yipeng Zhou, and Dah Ming Chiu. 2015. Analysis and Detection of Fake Views in Online Video Services. TOMM 11, 2s (2015).

[6] Claudia Czado. 2010. Pair-copula constructions of multivariate copulas. In Copula theory and its applications. Springer, 93-109.

[7] Arabin Kumar Dey and Debasis Kundu. 2009. Discriminating between the log-normal and log-logistic distributions. Commun. Stat. Theory Methods (2009).

[8] A. F. Costa, Y. Yamaguchi, A. J. M. Traina, C. Traina, Jr., and C. Faloutsos. 2015. RSC: Mining and Modeling Temporal Activity in Social Media. In KDD. ACM, 269-278.

[9] A.C. Favre, S. El Adlouni, L. Perreault, N. Thiémonge, and B. Bobée. 2004. Multivariate hydrological frequency analysis using copulas. Water Resources 40, 1 (2004).

[10] Arthur Gretton, Karsten M Borgwardt, Malte Rasch, Bernhard Schölkopf, and Alex J Smola. 2007. A kernel method for the two-sample-problem. In NIPS. 513-520.

[11] Charles N Haas, Josh Joffe, Mark S Heath, and Joseph Jacangelo. 1997. Continuous flow residence time distribution function characterization. F. of Env. Eng. 123 (1997).

[12] James Douglas Hamilton. 1994. Time series analysis. Vol. 2. Princeton Univ. Press.

[13] D. Juan, N. Shah, M. Tang, Z. Oian, D. Marculescu, and C. Faloutsos. 2017. M3A: Model, MetaModel and Anomaly Detection for Inter-arrivals of Web Searches and Postings. In DSAA. 341-350.

[14] Youngho Kim, Ahmed Hassan, Ryen W White, and Imed Zitouni. 2014. Modeling dwell time to predict click-level satisfaction. In WSDM. ACM, 193-202.

[15] J. C. Lagarias, J. A. Reeds, M. H. Wright, and P. E. Wright. 1998. Convergence properties of the Nelder-Mead simplex method in low dimensions. f. of Opt. 9, 1(1998).

[16] Chao Liu, Ryen W. White, and Susan Dumais. 2010. Understanding web browsing behaviors through Weibull analysis of dwell time. In SIGIR. 379-386.

[17] M. Marciel, R. Cuevas, A. Banchs, R. González, S. Traverso, M. Ahmed, and A. Azcorra. 2016. Understanding the detection of view fraud in video content portals. In $W W W$. IW3C2, 357- 368.

[18] José MR Murteira and Óscar D Lourenço. 2011. Health care utilization and self-assessed health: specification of bivariate models using copulas. Empirical Economics 41, 2 (2011).

[19] Thomas Nagler. 2018. VineCopula. https://cran.r-project.org/web/packages/ VineCopula/VineCopula.pdf. (2018).

[20] R. B. Nelsen. 2007. An introduction to copulas. Springer Science \& Business Media.

[21] Martin Ridout, Clarice GB Demétrio, and John Hinde. 1998. Models for count data with many zeros. In IBC, Vol. 19. IBS, 179-192.

[22] Neil Shah. 2017. FLOCK: Combating Astroturfing on Livestreaming Platforms. In WWW. 1083-1091

[23] TIME. 2016. Here'ÄŹs How Much Time Snapchat Users Spend on the App. http://time.com/4272935/snapchat-users-usage-time-app-advertising/. (2016).

[24] TIME. 2016. Instagram Just Hit the 500 Million User Mark. http: //time.com/money/4376329/instagram-users/. (2016).

[25] P. O. S. Vaz de Melo, L. Akoglu, C. Faloutsos, and A. A. F. Loureiro. 2010. Surprising Patterns for the Call Duration Distribution of Mobile Phone Users. In ECML-PKDD.

[26] Songhua Xu, Hao Jiang, and Francis Chi-Moon Lau. 2011. Mining user dwell time for personalized web search re-ranking. In IFCAI. AAAI.

[27] Xing Yi, Liangjie Hong, Erheng Zhong, Nanthan Nan Liu, and Suju Rajan. 2014. Beyond clicks: dwell time for personalization. In RecSys. ACM, 113-120.

[28] P. Yin, P. Luo, W.-C. Lee, and M. Wang. 2013. Silence is also evidence: interpreting dwell time for recommendation from psychological perspective. In KDD. ACM. 


\section{REPRODUCIBILITY}

\subsection{Data collection}

In this work, we collected a large dataset based on user engagement with Snapchat's Stories feature. We used publicly posted "My Story" snaps, which were made available to all Snapchat users (rather than the default "close friends" option) for privacy reasons. For our content modeling tasks, we collect viewing data associated with all such snaps posted in a 2 hours interval on a single day in May 2018. Furthermore, we collected all views associated with these Snaps (during the next $24 \mathrm{~h}$ hour span). We filtered the snaps that have duration $\leq 4$ seconds due to a discovered timer instability. Further, we truncated any views that appeared to persist longer than the media length; these are rare, but possible due to certain Snapchat app features like media long-presses.

For our viewer modeling task, we collected views to all Snaps over a 24 hour period. As previously mentioned, we conduct analysis only on Snaps with more than 100 views, and similarly filter out viewers who had viewed less than 100 Snaps during the associated timeframe. Due to privacy concerns and user agreements, the data is not shareable; however, we make our code developed for modeling and analysis available publicly.

\subsection{Copula preliminaries}

In this paper, we used copulas for aggregate modeling (Section 6). Copulas are a powerful statistical tool, which allow for scalable, parametric, approximate inference of multivariate distributions. As mentioned earlier, copulas are often used to model bivariate joint distributions, which is achieved by explicitly modeling the dependency structure given univariate marginals.

While bivariate copulas have demonstrated great empirical success in capturing dependencies via a variety of parametric forms, the number of generalized multivariate parametric copulas (for $>2$ variables) are highly limited and inflexible in preserving pairwise dependencies, resulting in poor estimation. Therefore, we use Vine copulas which model multivariate dependencies parametrically by flexibly modeling independent pairwise dependencies in high dimensions, effectively as multi-level trees. To elucidate, we give an example of a $C$-vine copula (one type of vine structure, which we used in this work) as follows:

Example. Consider three random variables $A, B, C$ and their corresponding marginal distributions $f_{A}, f_{B}, f_{C}$. In a $C$-vine, only one node is connected to all other nodes at each level tree. Thus, the first tree could be constructed by joining $A$ with $B$ and $C$, with the edges in the first tree becoming nodes in the second tree (see Figure 15). The associated joint distribution can be written as

$$
\begin{aligned}
& f_{V}(a, b, c)=f_{A} \cdot f_{B} \cdot f_{C} \\
& \text { [Nodes in Tree 1] } \\
& c_{A, B} \cdot c_{A, C} \\
& \text { [Edges in Tree 1] } \\
& c_{B, C \mid A} \\
& \text { [Edges in Tree 2] }
\end{aligned}
$$

\subsection{Implementation details}

We used Jupyter notebooks and Python to run all experiments on a high-memory, single-node Google Cloud compute engine instance.

For individual dwell time modeling (Section 5), we used the Python stat smodelsGenericLikeli hoodModel module, which enables general likelihood function maximization via builtin optimizers (we used Nelder-Mead). We used scipy.stats for parametric models

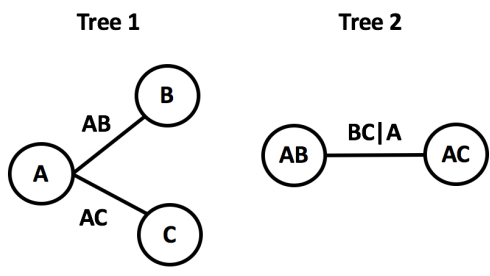

Figure 15: Illustration of $C$-vine copula structure: In Tree 1, marginals are used to fit bivariate copulas; these become nodes in Tree 2, between which another bivariate copula is fit.

of the discussed distributions (Log-logistic, Gamma, Weibull, Lognormal). We fixed the location parameter to 0 for all distributions while fitting, due to the uninterpretability of negative values in dwell times. We evaluated model fits using the KS_test functionality in scipy. stats to obtain test-statistic and $p$-values.

For aggregated dwell time modeling (Section 6), we used the $V$ ineCopula package in $R$ (well-documented and maintained). The $C$ vine dependency structure and parameter estimation was maximized according to log-likelihood. For computing the MMD test statistics to compare multivariate distributions, we used the kmmd package in $R$.

We make all our code available at https:/github.com/hemanklamba/ ModelingDwellTime.

\subsection{Generality}

Our work presents the largest to-date modeling, evaluation and analysis of multimedia dwell times to-date, and inferences are drawn from 300 thousand media samples, 24 million viewers and 273 million views. The data is a rich representation of multimedia engagement on the Snapchat platform in which visual multimedia is the predominant method of communication. Moreover, modeling inferences drawn were from sufficiently large sample sizes, suggesting that modeling inferences are consistent and accurate across a wide variety of user behaviors and content types. Though our work does not utilize data from other platforms for data availability and privacy reasons, we expect that the findings are representative of short-form visual multimedia at the least, but also have expected applicability to longer-form multimedia, given that most views to short-form content are even shorter, and decay with (super)exponential tails. 